\title{
UNA APROXIMACIÓN SINTÉTICA AL HACINAMIENTO SECTORIAL
}

\author{
Autores \\ Herman Torres Hernández \\ Carlos Ortiz Ochica \\ MBA - Facultad de Administración de Empresas - Universidad Santo Tomás
}

En aras de comprender el Hacinamiento Sectorial es necesario partir del enfoque general de donde se deriva, es decir, intentar una aproximación global de algunos conceptos que sirven como base de mercado para explicar sus raíces. Es por esta razón que se debe abordar el concepto de sector económico para ubicarse dentro de los límites espacio temporales de nuestra investigación, según el Departamento Nacional de Planeación (2000) (Banrepcultural) la actividad económica del país está dividida en partes, cuyos elementos poseen características, particularidades similares, que los hacen una unidad y a su vez las diferencian de otros grupos; su división y clasificación se efectúa partiendo de los procesos productivos que al interior de ellos se realizan.

Los sectores económicos a su vez contienen subdivisiones denominadas sectores estratégicos, los cuales se encuentran dentro de la clasificación CIIU del país. En los subsectores se encuentran aquellas empresas que son competidores directos ya que su desarrollo se limita y es afectado por las mismas variables (proveedores, compradores, bienes sustitutos y nivel de rivalidad).

\subsection{Rivalidad Empresarial}

La rivalidad entre las empresas, concepto desarrollado por Michael Porter dentro de sus cinco fuerzas del mercado (Porter, 1979), se puede definir como la disputa entre las empresas de un mismo sector económico, para posicionarse por encima de sus pares, y así adquirir una porción significativa del mercado utilizando diferentes tácticas como la disminución de precios, mejora o creación de productos, adquisiciones, etc.; situación que marca al interior de la empresa las necesidades de innovación para hacer frente a las estrategias de los competidores.

El nivel de rivalidad que se presente entre las empresas se intensifica en la medida en que se evidencie una mayor cantidad de los siguientes factores dentro del mercado: 
a) Un gran número de competidores dentro del mercado con un fuerte musculo financiero.

b) Bajo crecimiento del mercado el cual genera una mayor lucha entre los competidores por una pequeña porción de este.

c) Elevados costos fijos.

d) Poca diversificación.

e) Incrementos de la capacidad de producción dentro de una economía de escala.

f) Diversos competidores (nacionales e internacionales).

g) Altas barreras arancelarias.

\subsection{Isomorfismo}

Para comprender los diferentes fenómenos que enfrentan y afectan a las empresas, es indispensable saber que las empresas expresan, se desarrollan y evolucionan de acuerdo a la influencia generada por una persona o por un grupo de personas, a este hecho se le conoce como comportamiento laboral.

Se dice que las personas como entes pensantes, son conscientes que cuentan con un cerebro y lo reconocen como el centro de mando de todas las habilidades cognitivas, sin embargo, hoy en día está comprobado que dentro del cráneo se cuenta con "tres cerebros" conocidos como el cerebro básico reptiliano, el cerebro límbico mamífero y el cerebro humano neocortex.

¿Pero qué tiene que ver la clasificación de nuestro cerebro con el funcionamiento de las empresas?, sucede que en el cerebro conocido como el cerebro límbico, se cuenta con tres respuestas heredadas de la evolución propia del hombre, que aunque moderemos, siempre están presentes en nuestro actuar y se reflejan en cada acción o trabajo realizado por los seres humanos en su interacción con otros, estas tres respuestas límbicas son la parálisis, la huida y la lucha (Karlins, 2008).

El fenómeno conocido como Isomorfismo es una forma de expresión que ocurre en la respuesta límbica de la lucha, y se refiere a la imitación del comportamiento y estructura de dos seres, que buscan ser lo más parecidos posibles frente a su entorno. El nacimiento de este comportamiento 
ocurre cuando un ser humano imita las actitudes de otro ser humano o de un grupo social para ser parte de él y ser aceptado sin hacer uso consciente de la razón.

Este comportamiento se extiende a las empresas, que en su necesidad de lucha por el posicionamiento establecen normalmente políticas y estrategias basadas en la capacidad de imitar o copiar comportamientos exitosos de sus competidores y adaptarlos como parte del comportamiento propio y nativo de ellas mismas.

Cuando se revisa el isomorfismo con base en la sociología institucional, el cual busca explicar la tendencia de las empresas a imitarse en pro de ser aceptadas en el mercado de forma natural, apoyadas en el legado límbico de los directivos que guiados bajo la necesidad de la lucha organizacional, concepto que tiene como objetivo la explicación del fenómeno de la imitación; se genera el planteamiento de tres posibles orígenes, el isomorfismo coercitivo que obedece a la necesidad de la empresa para cumplir con estándares y procedimientos, el isomorfismo normativo que no es otra cosa diferente a la influencia de la experiencia profesional que homogeniza los estándares de conocimiento compartidos entre el personal que participa en las estructuras organizacionales, y por último el isomorfismo mimético o imitación de las prácticas exitosas del sector donde participa como jugador la empresa (DiMaggio \& Powell, 1983).

En el imaginario colectivo, que es un multiplicador del incremento del fenómeno del isomorfismo, se entiende como la imitación de la estrategia usada por una empresa para hacer de un proceso, servicio o producto un éxito, ya que al copiar esta estrategia o comportamiento se piensa que el mismo debe desencadenar un resultado de éxito parecido al inicialmente analizado. Sin embargo, este hecho lo único que desencadena es una convergencia estratégica en los sectores económicos.

Existen posturas adversas sobre el isomorfismo, a pesar de tener repercusiones negativas existen corrientes que defienden la imitación como estrategia de negocio. En estos planteamientos se destaca la posición de que la imitación es el acelerador para desarrollar nuevos productos, de tal manera que, también se encarga de incentivar y fomentar la innovación. Las empresas que imitan disminuyen el riesgo de incertidumbres comparadas con las empresas pioneras, dado esto se cuenta con mayor seguridad al éxito, logrando permanencia estable y sin mayores contratiempos. 
También, es necesario plantear que la mayoría de las reflexiones que se hacen sobre estrategia invitan a interpretar el entorno como un paso necesario hacia la comprensión de la realidad que rodea a la empresa (Rodriguez H. A., 2004). Repasando las diferentes corrientes de pensamiento usualmente reconocidas, se nota una inclinación hacia utilizar panoplias de instrumentos o herramientas orientadas a tal fin. Esto ha permitido a los estrategas y analistas sectoriales comprender, cada vez mejor, lo que sucede más allá de los límites de la organización. Sin duda, reflexionar sobre el medio que rodea a la empresa es un ejercicio interesante, contentivo de limitaciones, pero abundante en alternativas interpretativas, y tratar de captar la complejidad sobre lo que ocurre en los sectores, lo que se ha convertido en tema de investigación para una gran variedad de instituciones alrededor del mundo, en especial para la academia.

La realidad es que para que una empresa sea perdurable en el tiempo debe ejercer una fuerte planeación, lograr una ventaja competitiva real, la cual sea difícilmente imitable, y generar valor a sus clientes.

Mencionando el tema de ventaja competitiva sostenible, para la empresa existen dos ventajas alcanzables, la primera es la percepción exclusiva y la segunda la ventaja de costo más bajo (Puerta, 2004). En la ventaja de percepción exclusiva, la empresa busca el reconocimiento del consumidor, por lo cual este lo recompensa pagando mayores precios, su diferenciador es inigualable y su cadena de valor es difícilmente imitable, este tipo de ventaja genera clientes leales que hacen un portavoz de la marca para el consumo de servicios o productos. En la ventaja de costo más bajo, su objetivo es que los contrincantes identifiquen la empresa como el jugador con los mejores precios, la empresa debe tener una cultura orientada a la reducción de todos los costos en cada uno de sus procesos, una cultura organizacional orientada al ahorro.

Una cultura empresarial es el supuesto de la coordinación de los aspectos tanto sociales como culturales, es decir, la conexión entre sus formas establecidas de relación o nexos sociales, como de las interpretaciones subjetivas y actuaciones concretas en estas estructuras desarrolladas.

Debido a la velocidad del cambio en la actualidad, existe una mutación constante, la cual se está convirtiendo en un elemento consustancial a las culturas empresariales. La adaptabilidad es la 
respuesta de las organizaciones, claramente no respondiendo todas con la misma intensidad y potencia. (Caneda, DIRECCIÓN ESTRATÉGICA DE LA EMPRESA, 2015).

Para dar respuesta a la necesidad de supervivencia de las empresas y afrontar los diferentes fenómenos o enfermedades que deterioran los sectores e incrementan las tasas de mortalidad empresarial, se plantean tres elementos necesarios para favorecer la adaptación y el crecimiento empresariales, la primera es una capacidad transformada en actitud, conocida como la creatividad empresarial, la segunda es la forma de actuar para perseverar en el entorno, también llamada como innovación organizacional, y por último una estructura interna que involucre las dos anteriores, a esto se le denomina arquitectura organizativa innovadora. La creatividad empresarial es la habilidad que permite modificar las limitaciones autoimpuestas que tiene como objetivo la producción de ideas nuevas y valiosas tanto para la propia organización como para el negocio. En definitiva, supone no anclarse sólo en la producción basada en el ingenio o la ocurrencia para centrarse en el fomento de las capacidades de creación propias del ser humano con una vertiente netamente práctica, una vez resaltada la importancia que la creatividad tiene como factor de competitividad y supervivencia, se necesita su puesta en valor, su aplicación práctica en definitiva y esa es precisamente la función de la innovación.

Sobre el tema de innovación, Arie de Geus, antiguo vicepresidente de Planificación Estratégica de Shell y actual gurú de éxito, plantea que "la única ventaja competitiva verdaderamente sostenible es la habilidad para aprender más rápido que tus competidores”, no sólo es la necesidad de ser distintas que tienen las empresas para ser apreciadas por el público objetivo, sino que está también la necesidad de responder a sus necesidades a través de la mayor velocidad de presentación en los mercados.

En resumen, la innovación es la habilidad de transformar la creatividad en resultados tangibles y que aporten a la cifra de negocio así como a la cuenta de resultados, por último la arquitectura organizativa es la forma de repartir el trabajo y las responsabilidades entre los diferentes individuos que forman parte de las organizaciones, en especial las empresariales, con el fin de conseguir un objetivo común último, esto es, cómo organizarse para lograr los resultados empresariales marcados por la estrategia. Es, en resumen, la manera de estructurar las relaciones, 
plasmada habitualmente en un diseño gráfico denominado comúnmente organigrama. (Caneda, Dirección Estratégica Innovadora, 2010).

\subsection{Convergencia estratégica y erosión.}

Como se mencionó anteriormente, la convergencia estratégica es el resultado del isomorfismo presente en los actores dentro del mercado, ya que esta no es más que el desarrollo de estrategias similares entre las diferentes empresas que componen o pertenecen a un sector estratégico específico, la cual trae como consecuencia una erosión en la rentabilidad de los sectores estratégicos.

La convergencia estratégica se puede presentar en las empresas ya sea bien por el uso de los canales de distribución similares o por satisfacer de la misma manera las necesidades del mercado, la constante imitación entre las compañías genera una guerra de precios en el sector al cual pertenecen, lo que a su vez lleva a este a un futuro hacinamiento sectorial.

\subsection{Hacinamiento sectorial}

El hacinamiento sectorial genera la disminución de la tasa media de rentabilidad de un sector y se puede evidenciar en el mercado gracias a las similitudes en las acciones y estrategias que realizan las empresas de dicho sector. Para poder realizar este análisis se debe tener presente el siguiente algoritmo expresado en el libro "Análisis Estructural de Sectores Estratégicos": 
Gráfico 3. Algoritmo dos. Análisis de hacinamiento

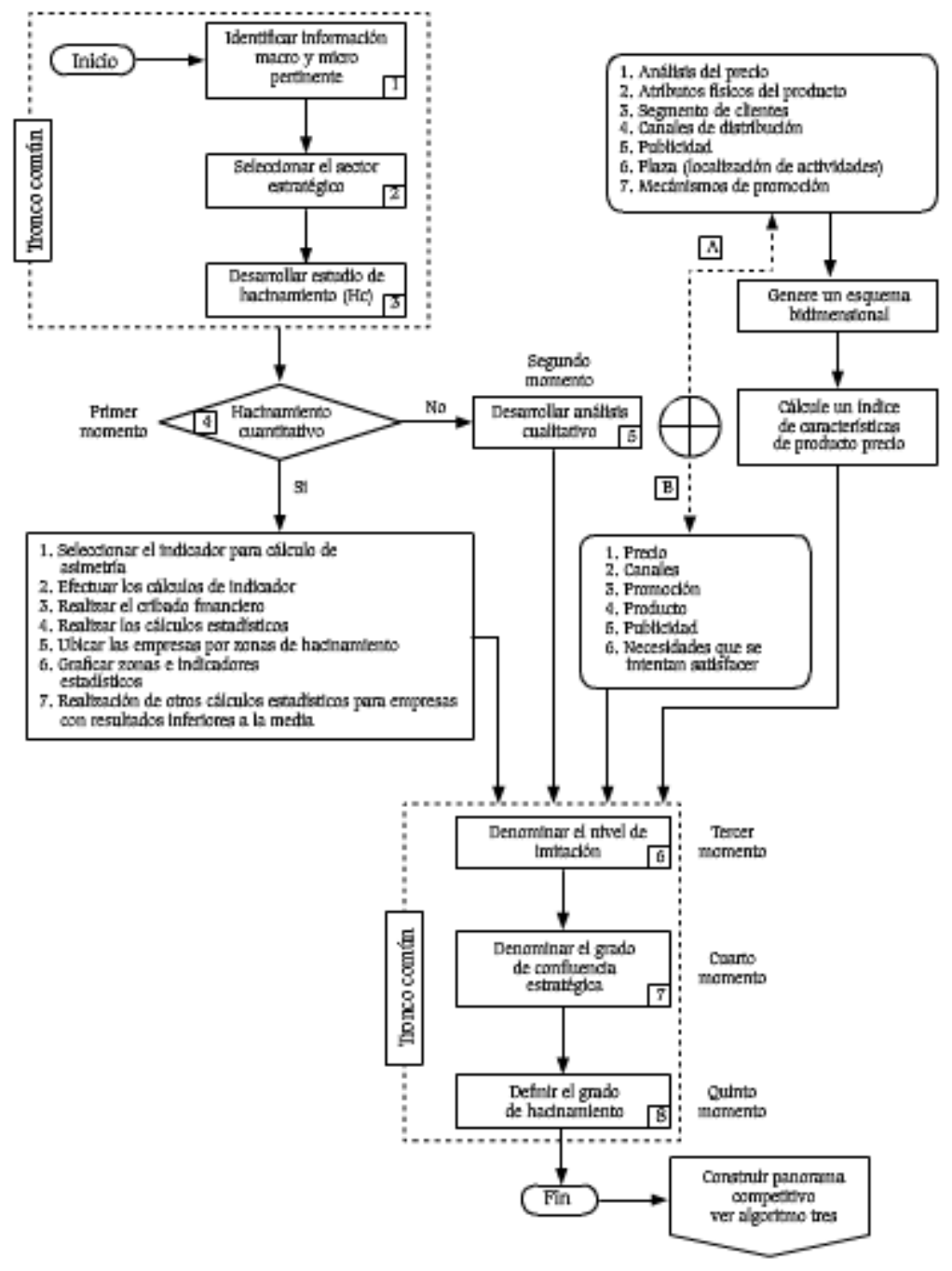

Fuente: Libro "Análisis Estructural de Sectores Estratégicos”

\subsubsection{Cuantitativo}

Para poder realizar un análisis completo del hacinamiento en un sector económico, se debe hacer una medición cuantitativa de este, la cual debe tener como base una valoración financiera donde se realiza un análisis estadístico de la información con elementos como la kurtosis, asimetría, diagramas de dispersión y el coeficiente de variación. Para lograrlo se deben realizar los siguientes pasos expuestos por Hugo 
Alberto Rivera Rodriguez en el documento "El hacinamiento, la enfermedad que los estrategas deben curar":

a) Selección del indicador para el cálculo de la asimetría: Se hace necesario el uso de un indicador cuantitativo para determinar si existe o no hacinamiento cuantitativo, este indicador no necesariamente debe ser financiero pero debe permitir evaluar el comportamiento del sector estratégico.

b) Efectuar los cálculos del indicador: cuando se cuenta con el indicador que ilustra el panorama del sector, se aplica los cálculos correspondientes a cada uno de las empresas que existen en el ecosistema del sector estratégico, el lapso de tiempo de los datos tomados no debe ser inferior a 5 años para cada una de ellas.

c) Cálculos estadísticos:

i. Cálculo y análisis del coeficiente de variación, el objetivo es identificar las empresas que se encuentran debajo de la media del indicador seleccionado

ii. Calculo de la desviación estándar, se dice que si al menos el $85 \%$ de las empresas del sector estratégico analizadas se encuentran en la media aritmética los resultados financieros son homogéneos, lo cual indica alto desempeño de algunas pocas empresas.

iii. Análisis de morbilidad y mortalidad, la morbilidad es la enfermedad que describe a empresas con incapacidad de obtener resultados superiores a la media del sector o incapacidad de obtener utilidad.

d) Ubicación de las empresas por zonas de hacinamiento:

i. Zona 1, empresas cuyo indicador se encuentra por encima del tercer cuartil

ii. Empresas ubicadas entre la media y el tercer cuartil

iii. Empresas ubicadas entre la mediana y la media

iv. Empresas ubicadas por debajo de la mediana o la media

v. Empresas con resultados negativos del indicador seleccionado 


\section{Zonas de hacinamiento}

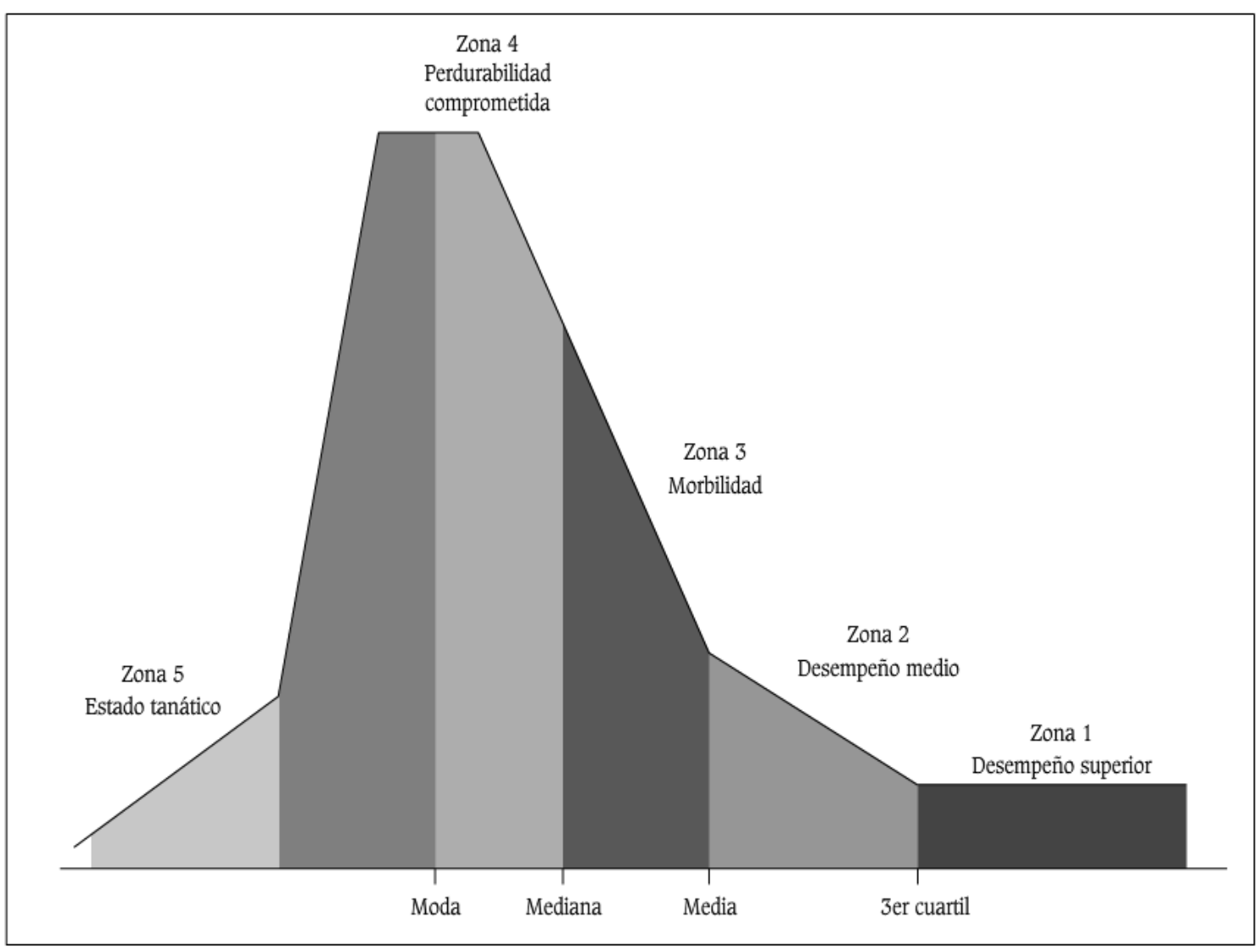

Fuente: Libro "Análisis Estructural de Sectores Estratégicos"

e) Generación de gráficas de las zonas e indicadores estadísticos: mediante los gráficos se espera mejorar la interpretación del analista para lograr conclusiones acertadas.

f) Análisis de datos: las empresas cuyo desempeño se encuentre por debajo del percentil 75 es necesario analizarlas y estudiarlas por separado.

\subsubsection{Cualitativo}

Nattermann (Nattermann) establece que para poder realizar un análisis cualitativo del hacinamiento sectorial completo se debe realizar una valoración de las características de producto, en pro de definir el posicionamiento dentro del sector de las compañías frente a sus competidores. 
Para hacerlo se deben tener presentes los atributos físicos del producto o servicio y el entorno económico en el que se desarrollan las compañías (canales de distribución, promoción y localización de actividades).

\subsubsection{Marketing Mix}

Para desarrollar un mejor análisis frente al hacinamiento sectorial se debe realizar una observación del marketing mix del mercado en el que se desenvuelven las empresas. Este se compone de los siguientes elementos:

\section{a) Precio:}

- Listado de precios del mercado.

- Descuentos ofrecidos a los consumidores.

- Periodos de pago.

- Medios de pago

\section{b) Producto}

- Tecnología

- Tiempos de entrega

- $\mathrm{I}+\mathrm{D}$

- Servicio post venta

c) Plaza

- Cobertura

- Infraestructura

- Cadena

\section{d) Promoción}

- Publicidad

- Promociones

- Relaciones Publicas 


\subsection{Panorama Competitivo}

Según Porter en 1987 (Rodriguez H. A., 2004), la empresa es contemplada como un conjunto de actividades que añaden valor al producto o servicio generado por la organización, cuya meta principal es diseñar, fabricar, comercializar y entregar el producto al consumidor final. De la misma forma es una herramienta estratégica que contribuye a la obtención de una ventaja competitiva donde el valor es aquella cantidad dispuesta a pagar por el comprador, por los atributos que ofrece una empresa en sus productos.

En la cadena de valor encontramos dos tipos de actividades: Actividades primarias y actividades de apoyo. "Las primarias son aquellas que contribuyen a la creación física del producto o servicio, su venta y transferencia al cliente y sus servicios postventa” (Porter M., 2002, pág. 68), las actividades de apoyo añaden valor a través de importantes relaciones a la empresa en colaboración con las actividades primarias. La ventaja competitiva establece el papel primordial en el desarrollo y crecimiento organizacional de las empresas. Con base en el planteamiento anterior, las organizaciones se deben enfocar en el desarrollo de actividades empresariales para lograr un valor agregado que les permitan desarrollar productos y/o servicios perdurables en el tiempo, que no se vean afectados por las enfermedades que el sector pueda llegar a adquirir.

\section{Cadena de Valor y Cadena de Suministros}

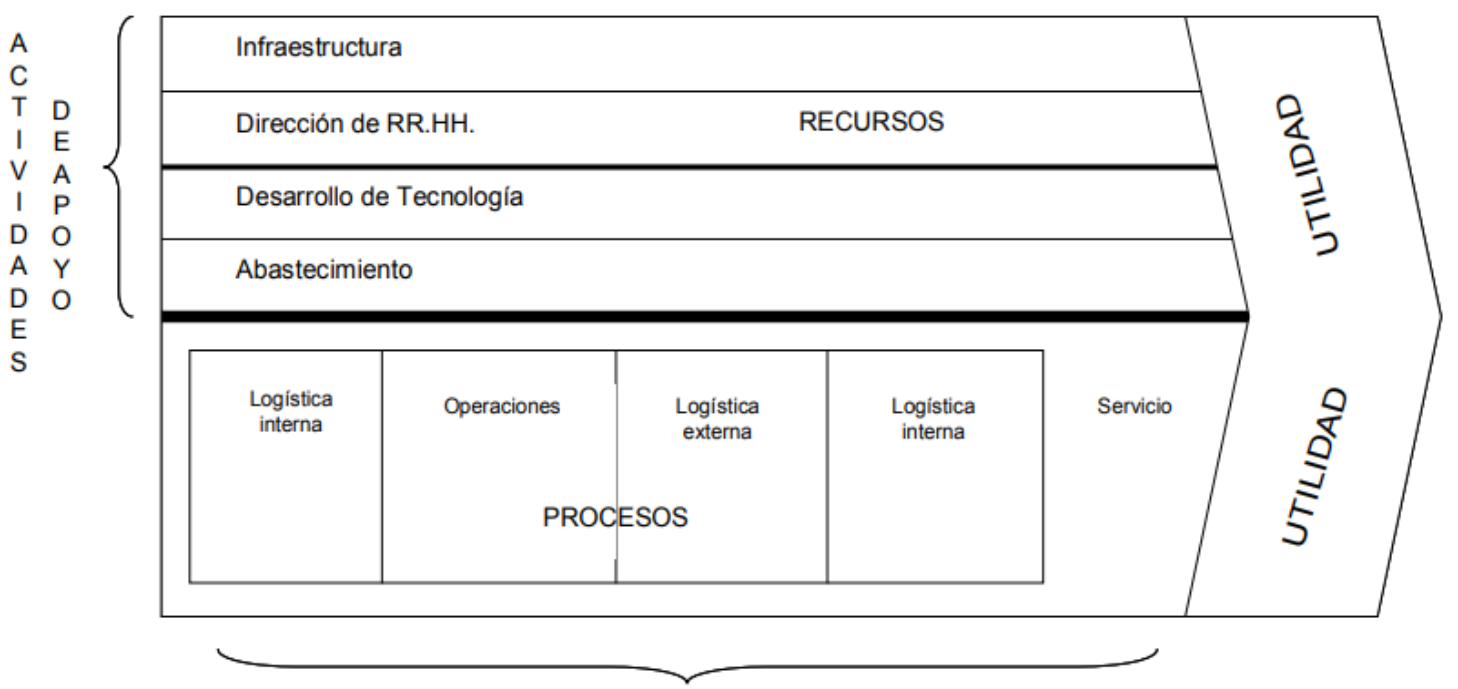

ACTIVIDADES PRIMARIAS 
El panorama competitivo es la técnica empleada para determinar las manchas blancas que existen en el sector de estudio. Una mancha blanca es el nombre que reciben las oportunidades que no se cubren en la relación producto-mercado de las actuales unidades de negocios, en otras palabras, los espacios de mercado que se encuentran débilmente atendidos o son inatendidos totalmente. Esto permite contar con una visión permanente de todas las posibilidades presentadas a un grupo de estrategia, cuyo fin es la explotación y maximización de las nuevas variedades, las nuevas necesidades o los nuevos canales de distribución. Según Porter (Puerta, 2004) el levantamiento del panorama permite lograr una mejor posición estratégica dentro del sector que surge de tres fuentes diferentes las cuales son posicionamiento basado en variedad, necesidad y canales de acceso.

La amplitud o estrechez del panorama está relacionada directamente con los competidores: es decir, un panorama amplio implica tener en cuenta un amplio rango de segmentos de productos y compradores, pero en otros se hace necesario requerir de alianzas, integraciones y de productos complementarios para poder ser competitivos. Como existen muchas formas de segmentar, las estrategias, a su vez pueden contener un poco de panorama amplio, como de uno más estrecho (Rodríguez \& Puerta, 2008)

\subsubsection{Estructura del Panorama Competitivo}

La realización del panorama competitivo, brinda un apoyo frente a las decisiones estratégicas de las empresas ya que se puede generar una radiografía del sector, con la cual se llega a evidenciar la totalidad de posibilidades estratégicas para dichas empresas. Para llegar a identificar el panorama sectorial se puede hacer uso de una matriz $\mathrm{T}$ o de un paralelepípedo estratégico, los

cuales necesitan para su construcción de tres vectores específicos: necesidades, canales y variedades. 


\section{Estructura del panorama competitivo}

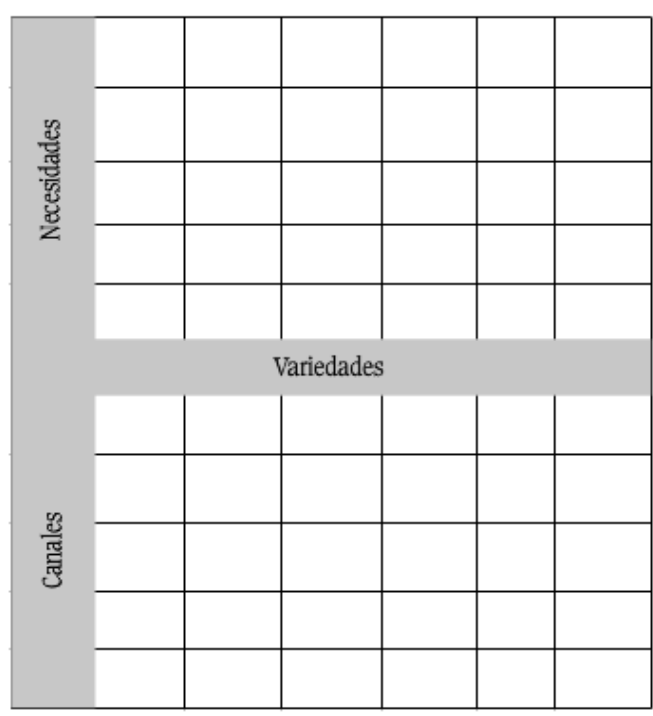

1 a. Matriz "T"

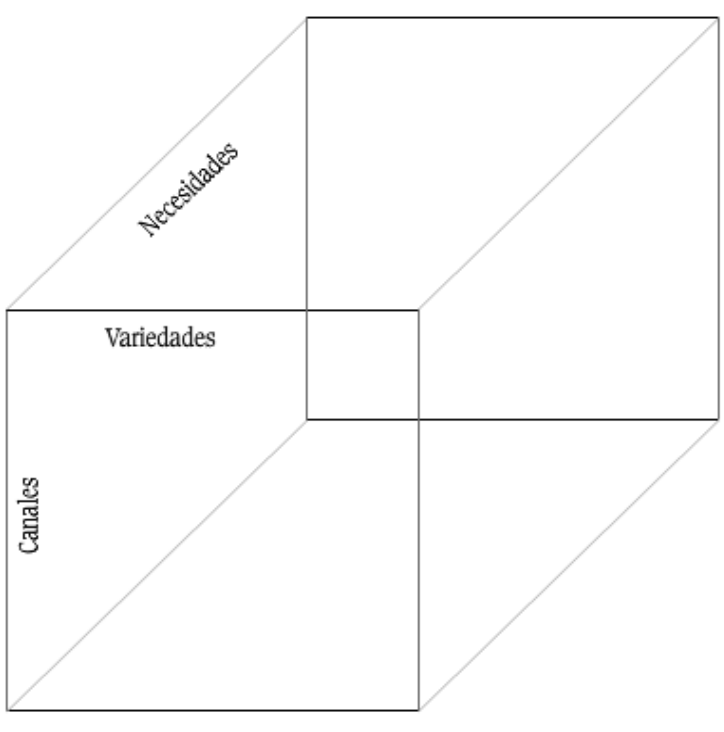

1 b. Paralelepípedo

Fuente: Libro "Análisis Estructural de Sectores Estratégicos"

a) Necesidades: Este vector debe contener la totalidad de las necesidades que se satisfacen en el sector de estudio. Las necesidades se pueden clasificar en las necesidades del usuario (consumidor final) y necesidades del canal (intermediario). Adicional a esta clasificación, también se puede tener presente la pirámide de necesidades de Maslow, en la cual, las necesidades se dividen en cinco grupos: necesidades fisiológicas; necesidades de seguridad; necesidades sociales; necesidades de estima; y necesidades de realización. (Pichère, Cadiat, \& Serra, 2026).

b) Variedades: En este se establece la totalidad de los productos o servicios que se ofrecen en el sector estratégico que se está estudiando.

c) Canales de distribución: Este vector está compuesto por los medios a través de los cuales el cliente adquiere los productos o servicios ofrecidos en el mercado.

Se establecen estos tres vectores teniendo en cuenta que según Porter (Puerta, 2004), para que una empresa logre tener un posicionamiento en el mercado debe tener presente tres fuentes, la variedad, las necesidades y el segmento de los clientes, fuentes que están directamente relacionadas con dichos vectores. Adicionalmente tanto la publicidad como el precio son excluidos al momento de establecer los vectores principales ya que según estudios realizados en 
el libro "el hacinamiento, la enfermedad que los estrategas deben curar", estos son efectos de la convergencia que se pueda presentar en un sector.

El modelo básico también conocido como panorama bidireccional define de forma cualitativa y cuantitativa los factores que se deben considerar en la estructuración de un sector específico. De esta forma se ha llegado a un modelo en el que el interés es hacer énfasis en los aspectos competitivos de perdurabilidad de una empresa del sector en el cual se encuentra. La metodología que emplea este enfoque se basa en una organización bidimensional de variedades vs. Parámetros (necesidades y canales) asociados a organizaciones presentes en el sector (Johann Heinz Martínez Huartos, 2011).

\section{Modelo básico del panorama competitivo}

\begin{tabular}{|c|c|c|c|c|c|c|c|}
\hline \multirow{4}{*}{ हू } & & & & & & & \\
\hline & & & & & & & \\
\hline & & & & & & $\mathrm{C}_{j}$ & \\
\hline & & & & & & & \\
\hline \multirow{2}{*}{ Variedades } & \multicolumn{3}{|c|}{ Producto 1} & & \multicolumn{3}{|c|}{ Producto n } \\
\hline & Org 1 & Org 2 & Org m & $\cdots$ & Org 1 & Org 2 & . 0 Org k \\
\hline
\end{tabular}

\subsubsection{Levantamiento del panorama competitivo}

Para facilitar el proceso de construcción del panorama competitivo, Rodríguez, H. A., \& Puerta, L. F en su libro "el hacinamiento, la enfermedad que los estrategas deben curar", establecen el siguiente algoritmo: 


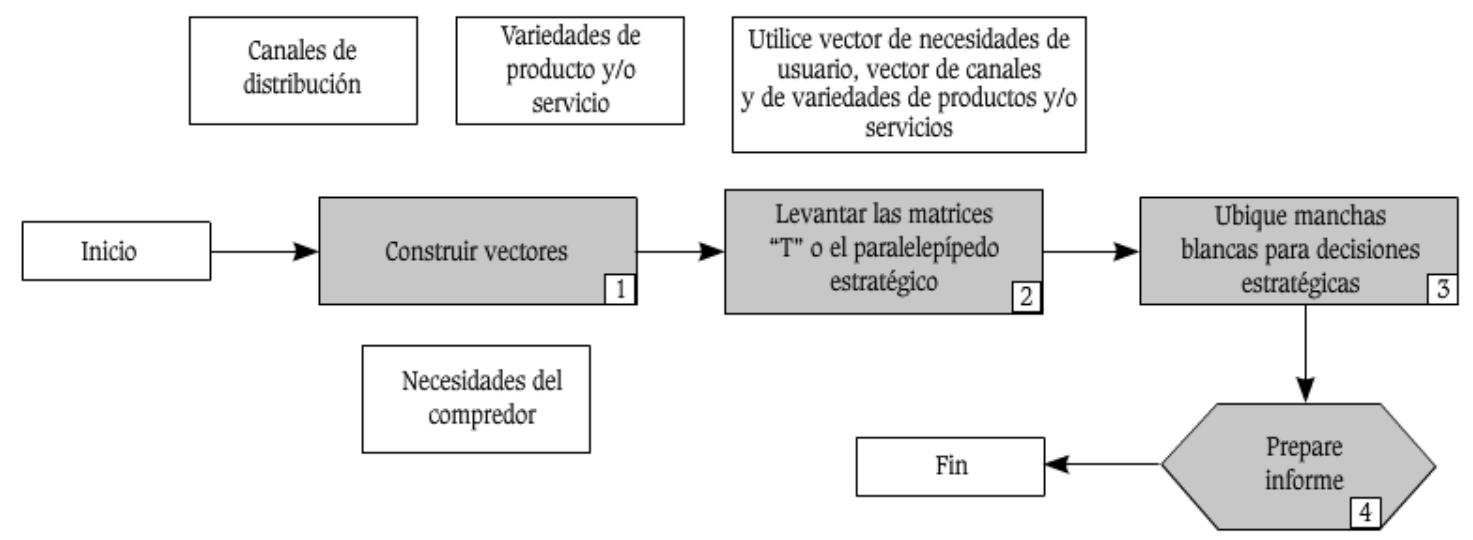

Fuente: Libro “Análisis Estructural de Sectores Estratégicos”

De este modo para la construcción de los vectores es necesario realizar encuestas a los expertos sobre los productos o servicios, características diferenciadoras, Usuarios, necesidades que se satisfacen y canales de distribución, acto seguido y contando con los vectores, se procede con la elaboración del panorama estratégico por medio de la matriz T o el paralelepípedo estratégico, en este punto es necesario identificar las empresas a analizar "rivales o competidores directos" y diligenciar las casillas identificando las necesidades, variedades y canales. Ahora se procede con la ubicación de las manchas blancas para lo cual se realiza el cruce de información, se identifica si diversas empresas satisfacen una necesidad propia del entorno de la misma forma, se determina si existen empresas con ventaja competitiva y se describen los espacios en blanco no explotados, para finalizar se genera el informe de estado del sector y las recomendaciones.

\subsubsection{Matriz T}

Esta alternativa para el levantamiento del panorama competitivo, ayuda a comprender que canales de distribución son los utilizados por los competidores de una empresa dentro de un sector estratégico o cuales son las necesidades específicas que están siendo satisfechas dentro de este.

Como se evidencia en la siguiente gráfica en la matriz se establecen las necesidades de los clientes del sector estratégico, los canales de distribución, la variedad de productos o servicios ofrecidos y las empresas que se encuentran dentro de este sector. 


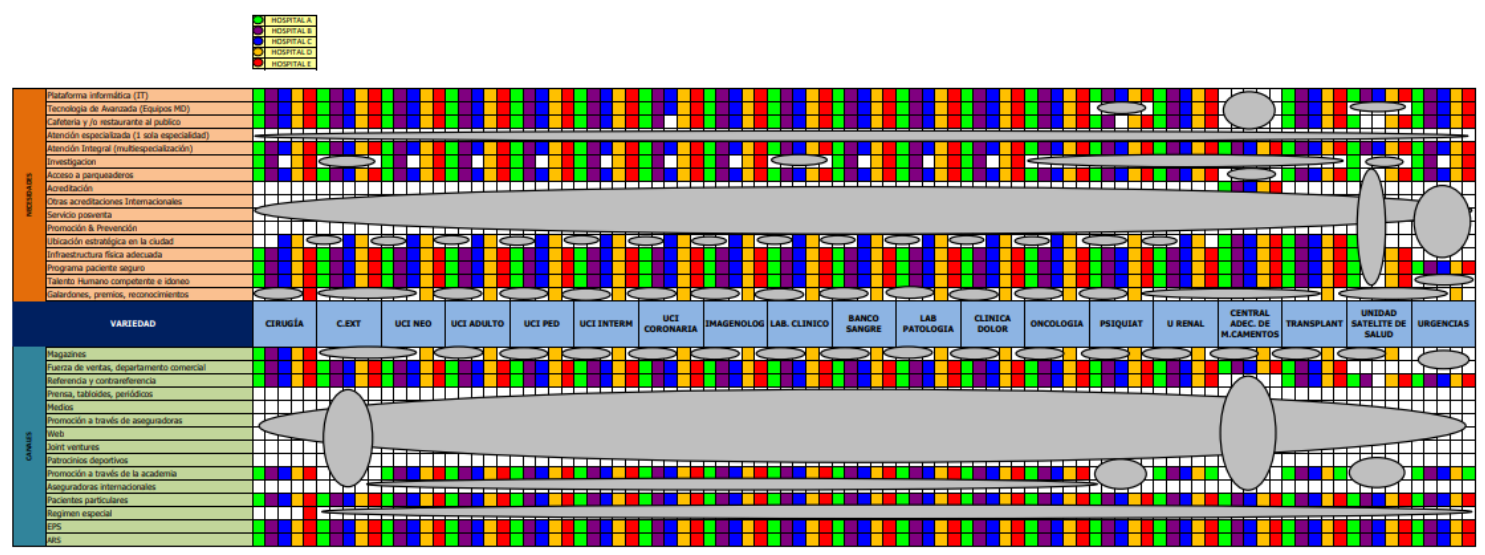

Fuente:http://repository.urosario.edu.co/bitstream/handle/10336/4304/Anexo_1_Matriz_T.pdf?se quence $=2$

a) Relación variedades- necesidades: Los espacios vacíos que se encuentren al momento de relacionar las variedades y las necesidades son mercados libres para atacar, es decir nuevas oportunidades de mercado.

Por otro lado, aquellos espacios en los cuales se ubiquen totas las empresas satisfaciendo las mismas necesidades con la misma variedad de productos o servicios, son mercados en los cuales se puede llegar a competir por precio y a su vez, generar una erosión en la rentabilidad de las empresas que allí se ubiquen.

b) Relación variedades - canales: Si al relacionar las variedades con los canales de distribución se encuentra ubicadas todas las empresas en un solo punto, se puede concluir que este es el canal más utilizado en mercado para acercarse al cliente final, pero si se evidencia un espacio vacío esta es una oportunidad para las empresas de atacar el mercado por otro canal en el que no se tendrá un alto nivel de competencia, esto siempre y cuando genere una valor agregado a la empresa.

Al realizar la matriz $\mathrm{T}$, se deben responder preguntas tales como:

- ¿Qué productos están satisfaciendo las necesidades del sector?

- ¿Todas las empresas tiene el mismo target?

- ¿Qué canales están siendo utilizados en el mercado? 
- ¿Qué segmentos de mercado están siendo atendidos por las empresas que están dentro de un sector estratégico?

\subsubsection{Paralelepípedo estratégico}

Al realizar este se puede tener un análisis más directo de lo que ocurre entre la necesidad del mercado y las variedades de productos o servicios ofertados para su satisfacción.

Primero se deben identificar la totalidad de los vectores y sub vectores que componen el caso analizado, por ejemplo: en el sector de los lácteos se presentan tres necesidades, tres canales y tres variedades, dando un total de 27 subvectores. Así mismo, cada vector y subvector tiene un peso relativo diferente frente a los demás, por ejemplo necesidades pesa un $50 \%$, canales un $25 \%$ y variedades un $25 \%$.

Después se deben definir los paralelepípedos y subparalelepípedos dentro del sector estratégico; la cantidad de los subparalelepípedos sale de la multiplicación de los elementos de cada uno de los vectores identificados anteriormente. Por ejemplo, $3 \mathrm{~N}$ x $3 \mathrm{~V}$ x $3 \mathrm{C}=27$ subparalelepípedos. Se debe tener presente que cada uno de los subparalelepípedos o cubos tiene un tamaño diferente ya que el peso relativo de estos es diferente.

Para finalizar se debe identificar y ubicar cada una de las empresas que se desarrollan en cada subparalelepípedo.
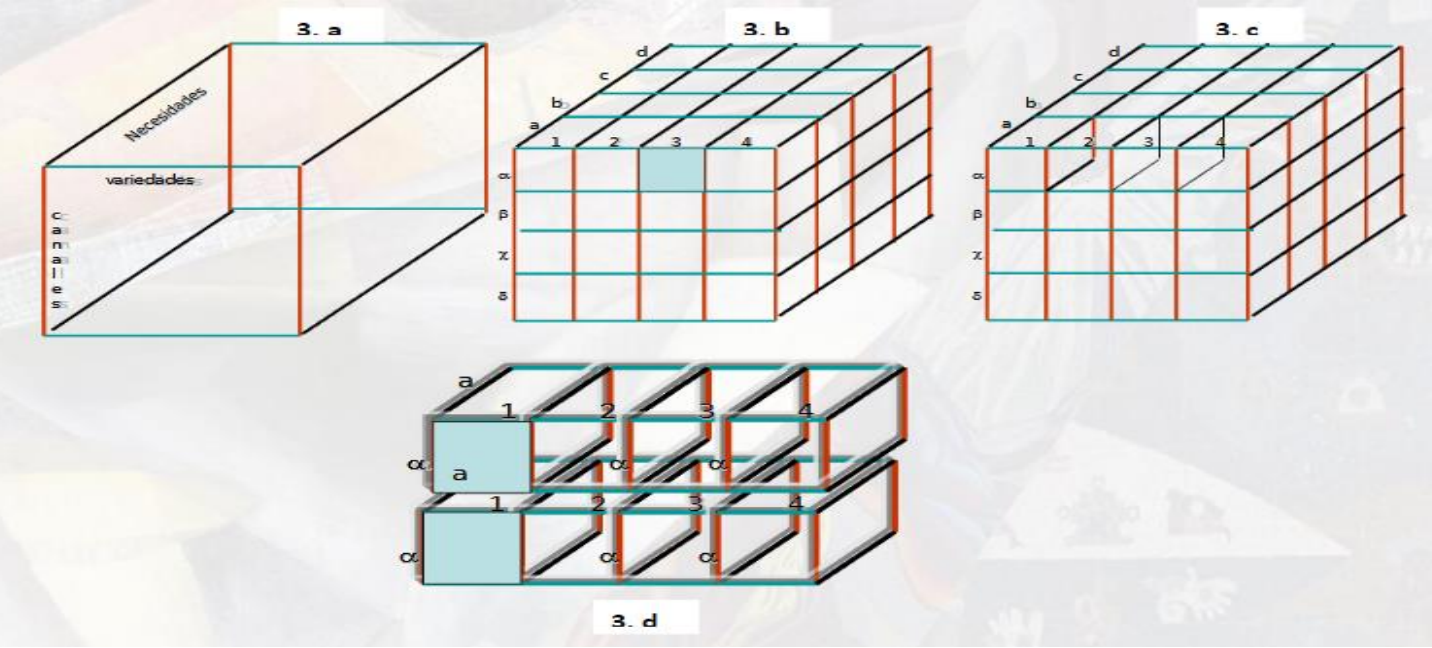

Fuente: Researchgate.net 


\section{Bibliografía}

ISOMORFISMO VS. EFICIENCIA EN EL ANÁLISIS ORGANIZACIONAL. (Enero de 1999). Empresa y Humanismo., págs. p137-144.

Universida del Rosario. (2012). Obtenido de http://repository.urosario.edu.co/bitstream/handle/10336/4304/Anexo_1_Matriz_ T.pdf? sequence $=2$

Caneda, M. C. (2010). Dirección Estratégica Innovadora. Coruña: Netbiblo, S.L.

Caneda, M. C. (2015). DIRECCIÓN ESTRATÉGICA DE LA EMPRESA. La coruña: IFFE Business School.

DiMaggio, P. J., \& Powell, W. W. (1983). The Iron Cage Revisited Institutional Isomorphism and Collective Racionality. American Sociological Review, 48(2), 147-160.

Johann Heinz Martínez Huartos, H. A. (2011). Adquisición de ventajas competitivas mediante la generación de un territorio estratégico. Mexico.

Karlins, J. N. (2008). El cuerpo habla. Editoria Sirio, S.A.

Marín-Idárraga, D. A. (oct-dic2013). La conformación del currículo en Administración: un estudio desde el isomorfismo institucional. Estudios Gerenciales, Vol. 29 Issue 129, p466-475. 10p.

Mintzberg, H. (s.f.). Planning on the left side and manging on the right. Harvard Business Review.

Nattermann, P. M. (s.f.). Competitor Behavior in response to new entry: The Case of the German mobile phone market 1986-1998. Competitor Behavior in response to new entry: The Case of the German mobile phone market 1986-1998.

Pichère, P., Cadiat, A.-C., \& Serra, M. M. (2026). La pirámide de Maslow: Conozca las necesidades humanas para triunfar.

Puerta, L. F. (2004). Interpretando a Porter. Bogota: Centro Editorial Universidad del Rosario.

researchgate. (s.f.). Obtenido de https://www.researchgate.net/publication/319911401_CUANTIFICACION_DE_ LAS_NUEVAS_OPORTUNIDADES_DE_MERCADO_A_TRAVES_DE_UN_ PANORAMA_COMPETITIVO_TRIDIMENSIONAL

Rodriguez, H. A. (2004). El hacinamiento, la enfermedad que los estrategas deben curar. Rodriguez, H. A. (2004). El hacinamiento, la enfermedad que los estrategas deben curar. Rodríguez, H. A., \& Puerta, L. F. (2008). Análisis estructural de sectores estratégicos. En H. A. Rodríguez, \& L. F. Puerta, Análisis estructural de sectores estratégicos (pág. 87). Colombia: Universidad del rosario. 